\title{
Proper hepatic pedicle clamping during hepatectomy is associated with improved postoperative long-term prognosis in patients with AJCC stage IIIB hepatocellular carcinoma
}

\author{
Xiaoqiang $\mathrm{Li}^{1,2, *}$, Shuang Liu ${ }^{1,2, *}$, Hui $\mathrm{Li}^{1,2, *}$, Lei Guo ${ }^{1,2}$, Bo Zhang ${ }^{1,2}$, Zhenhai Lin ${ }^{3}$, \\ Jubo Zhang ${ }^{4}$, Qinghai Ye ${ }^{1,2}$ \\ ${ }^{1}$ Liver Cancer Institute and Zhongshan Hospital, Fudan University, Shanghai 200032, P.R. China \\ ${ }^{2}$ Key Laboratory of Carcinogenesis and Cancer Invasion, Ministry of Education, Shanghai 200032, P.R. China \\ ${ }^{3}$ Department of Hepatic Surgery, Shanghai Cancer Center, Fudan University, Shanghai 200032, P.R. China \\ ${ }^{4}$ Department of Infectious Diseases, Huashan Hospital, Fudan University, Shanghai 200040, P.R. China \\ "These authors contributed equally to this work \\ Correspondence to: Qinghai Ye, e-mail: ye.qinghai@zs-hospital.sh.cn \\ Jubo Zhang, e-mail: zhang.jubo@zs-hospital.sh.cn
}

Keywords: hepatocellular carcinoma, hepatic pedicle clamping, hepatectomy, tumour stage, prognosis

Received: January 17, 2016

Accepted: February 28, 2016

Published: March 24, 2016

\section{ABSTRACT}

Intermittent hepatic pedicle clamping (HPC) is often performed during hepatectomy. Whether it affects the long-term prognosis of hepatocellular carcinoma (HCC) patients is still controversial. This study evaluated the impact of HPC in patients with different stages of HCC. The study included 1401 patients who underwent hepatectomy in the primary cohort with 129 AJCC stage IIIB HCC patients; there were 80 AJCC stage IIIB HCC patients in the validation cohort. In each cohort, patients were placed in the long-term HPC (LTHPC) group or the short-term HPC (STHPC) group based on the cut-off time of HPC estimated by the receiver-operating characteristic (ROC) curve. Although HPC did not show significant effects on the prognosis of stage I-IIIA HCC patients in the primary cohort, 1-, 3-, and 5-year overall survival (OS) and recurrence-free survival (RFS) rates of stage IIIB HCC patients who received LTHPC (HPC time > 12 minutes) were significantly higher than those with STHPC (HPC time $\leq 12$ minutes or received no HPC), similar in the validation cohort. Multivariate analysis demonstrated HPC time was an independent protective factor for RFS and OS in stage IIIB HCC patients. Herein, we report that proper HPC improved the postoperative prognosis of stage IIIB HCC patients and served as an independent protective factor.

\section{INTRODUCTION}

Primary liver cancer, $75-80 \%$ of which is hepatocellular carcinoma (HCC), is the fifth most common cancer and the second leading cause of cancer death in males worldwide [1]. Eastern Asia and sub-Saharan Africa are regions with the greatest incidence of HCC with a predominance of the male over the female gender (3/4:1) in the Asia-Pacific region, over 20 per 100,000 individuals [2]. Current guidelines do not recommend liver transplanting for HCC patients outside the Milan criteria because of the long time waiting for donor and absence of sufficient available evidence [3]. Percutaneous local ablative techniques are the choices in patients who are not candidates for surgery [4]. Liver resection is still the main curative treatment for HCC patients [5-7]. Unfortunately, even after curative hepatectomy, the postoperative recurrence-free survival (RFS) and overall survival (OS) rates were still unsatisfactory, with a 5-year recurrence rate of approximately $70 \%$ after hepatectomy [8]; prognosis is also poor in patients with advanced HCC (i.e., stage IIIB HCC). Therefore, how to improve the prognosis of HCC patients is still clinically concerning. Recently, several animal studies reported that hepatic ischemia-reperfusion ( $/ / R)$ injury due to hepatic pedicle clamping (HPC) caused accelerated tumour growth and promoted metastases [9-12]. Whether this is the case in HCC patients is still controversial, but intermittent HPC is widely used to reduce blood loss during hepatectomy [13]. In 2005, Makino et al. reported the prognostic benefit 
of selective portal vein occlusion relative to total portal vein occlusion during hepatic resection for HCC patients [14]. Yang et al. also found that selective main portal vein occlusion could minimize the risk of recurrence after curative resection of $\mathrm{HCC}$ [15].

Given the conflicting data, the aim of this study was to explore whether the HPC could improve the recurrencefree and overall survival of $\mathrm{HCC}$ patients in a large, consecutive single institution cohort.

\section{RESULTS}

\section{HCC patients with or without HPC did not show significant differences in prognosis}

By the end of this study, 728 of 1401 patients had developed tumour recurrence $(52.0 \%)$ (stage I: $45.7 \%$, stage II: $56.4 \%$; stage IIIa: 76.6\%; stage IIIb: 58.9\%) and 688 patients $(49.1 \%)$ (stage I: $36.1 \%$; stage II: $57.3 \%$; stage IIIa: $76.6 \%$; stage IIIb: $81.4 \%$ ) had died. The $1-$, 3-, and 5-year RFS and OS rates in patients without HPC during hepatectomy were relatively higher than those with HPC $(76.3 \%, 54.8 \%, 47.2 \%$ versus $72.5 \%$, $52.7 \%, 47 \%$; $88.2 \%, 63.7 \%, 53.9 \%$ versus $82.1 \%, 61.4 \%$, $53.8 \%$ ), but with no significant differences. To find the best cut-off of HPC, rather than that simply defined as given HPC or not, we used the ROC curve (described in Materials and Methods). The best cut-off of HPC was 4 minutes for all patients of the primary cohort. Then, we redistributed the patients into the short-term hepatic pedicle clamping group (STHPC group; HPC time $\leq 4$ min or without HPC; $n=993$ ) and the long-term hepatic pedicle clamping group (LTHPC group; HPC time $>4$ $\min ; n=408)$. The clinicopathological characteristics of the two groups are presented in Table S1. There were no significant differences in age, gender, HBsAg status, serum AFP concentration, multiplicity, tumour capsule, and Edmondson-Steiner grading. Lower rate of liver cirrhosis, bigger tumour size, and fewer stage I and stage II HCC patients were observed in the LTHPC group compared with the STHPC group $(P=0.005, P<0.001$, $P=0.002$; Table S1). However, RFS and OS showed no significant differences between the STHPC and LTHPC groups $(P>0.05$, data not shown).

\section{Prognosis improvement for stage IIIB HCC patients who received LTHPC in the primary cohort}

As proposed, HCC patients with different clinical AJCC TNM stages might respond differently to HPC during surgery. ROC curve analysis was performed for patients with stage I, II, IIIA, and IIIB in the primary cohort, and the best cut-off times were 11, 13, 14, and 12 minutes, respectively. Whether HPC lasted longer than the cut-off time had no significant effects on RFS $(P=0.324$,
$0.219,0.32)$ and $\mathrm{OS}(P=0.344,0.544,0.085)$ of patients with stage I, II, and IIIA HCC (Figure S1). However, stage IIIB patients who received HPC for more than 12 minutes (LTHPC group, $n=30$ ) had a better prognosis than those who received HPC for 12 minutes or less or those who did not receive HPC (STHPC group, $n=99$; Figure 1). The clinicopathological characteristics of the two groups of stage IIIB patients are summarized in Table S2. There were no significant differences in these characteristics (Table S3), except that the proportion of patients with higher serum AFP concentration is larger in the STHPC group compared with the LTHPC group $(P=0.047)$.

In stage IIIB patients of the primary cohort with the median follow-up 14.0 months, the median RFS time of LTHPC group was significantly longer than that of the STHPC group (24.0 months vs. 13.0 months; $P=0.006$ ), and 1-, 3-, and 5-year RFS rates of the LTHPC group were higher than those of the STHPC group $(67.73 \%$ vs. $49.87 \%, 52.68 \%$ vs. $27.27 \%, 52.68 \%$ vs. $19.52 \%$, respectively). Consistently, the median OS time was 34.28 months in the LTHPC group, which was longer than that (20.86 months) in the STHPC group $(P=0.046$; Figure 1$)$. The 1-, 3-, and 5-year OS rates of the LTHPC group were higher than those of the STHPC group $(63.33 \%$ vs. $53.30 \%, 36.67 \%$ vs. $17.33 \%, 36.67 \%$ vs. $12.45 \%$, respectively). Univariate analysis showed that the HPC time $(P=0.007)$, tumour capsule $(P=0.025)$, and tumour size $(P=0.029)$ were associated with RFS. Multivariate analysis using the Cox model demonstrated that the HPC time $(P=0.005, \mathrm{HR}=0.41,95 \% \mathrm{CI}=0.220-0.765)$ and tumour capsule $(P=0.013, \mathrm{HR}=2.019,95 \%$ $\mathrm{CI}=1.157-3.523)$ were independent prognostic factors for RFS (Table 1). For OS, univariate analysis showed that the only significant protective factor was HPC time $(P=0.043$; Table 1).

\section{Verified prognosis improvement of stage IIIB patients who received LTHPC in a validation cohort}

To verify the importance of proper HPC during hepatectomy, another cohort of 80 patients with stage IIIB HCC was collected, with the median follow-up 12.6 months. These patients were also divided into the LTHPC group (HPC time $>12$ minutes, $n=43$ ) and the STHPC group (HPC time $\leq 12$ minutes or without HPC, $n=37$ ). The clinicopathological characteristics and surgical outcomes of these two groups in the validation cohort are summarized in Table S4 and Table S5, and no significant differences were observed between the two groups. The median RFS time of the LTHPC group was significantly longer than that of the STHPC group (12.5 months vs. 7.3 months, $P=0.030$; Figure 2). The 1-, 3-, and 5-year RFS rates of the LTHPC group were higher than that of the STHPC group (53.79\% vs. $29.08 \%, 34.33 \%$ vs. $10.90 \%, 22.89 \%$ vs. $10.90 \%$, respectively). The median OS 
time was 19.4 months in the LTHPC group, which was also longer than that of the STHPC group (8.5 months, $P=0.005$; Figure 2). The 1-, 3-, and 5-year OS rates in the LTHPC group were higher than those in the STHPC group (76.06\% vs. $37.84 \%, 34.29 \%$ vs. $10.81 \%, 28.58 \%$ vs. $10.81 \%$, respectively). Univariate analysis revealed that only HPC time was a protective factor affecting RFS $(P=0.030$; Table 2). Using multivariate analysis for OS, HPC time is the only independent protective factor $(P=0.019, \mathrm{HR}=0.535,95 \% \mathrm{CI}=0.317-0.904$; Table 2$)$.

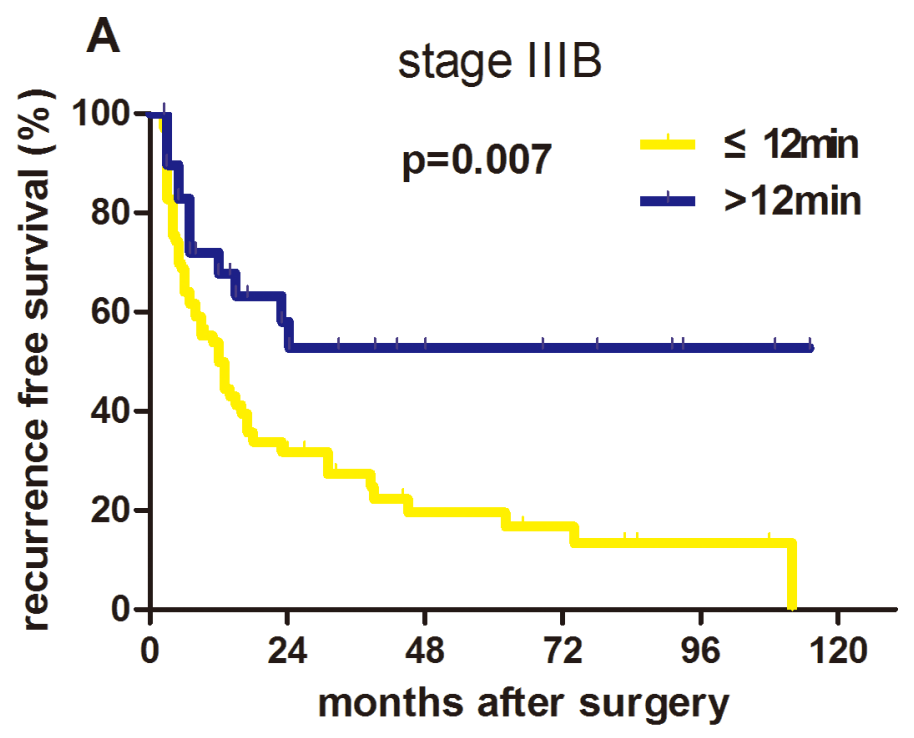

No. of patients at risk

HPC time (min)

$\begin{array}{llllll}\leq 12 & 99 & 16 & 8 & 6 & 3 \\ >12 & 30 & 12 & 8 & 6 & 3\end{array}$

B stage IIIB

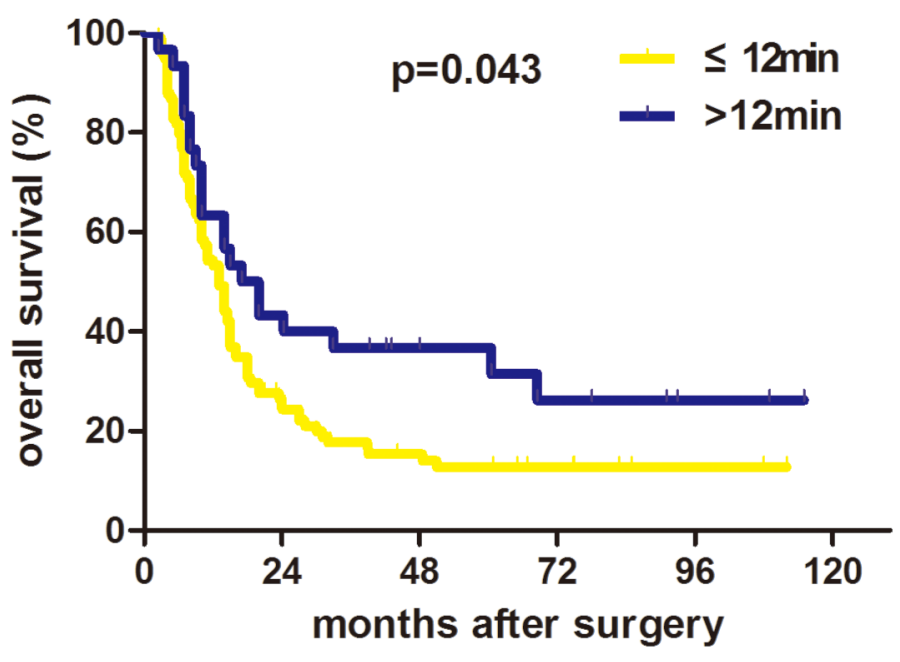

No. of patients at risk

HPC time (min)

$\begin{array}{lllccc}\leq 12 & 99 & 24 & 13 & 8 & 4 \\ >12 & 30 & 15 & 9 & 6 & 3\end{array}$

Figure 1: Proper HPC application benefited the prognosis of stage IIIB HCC patients in the primary cohort. Patients with stage IIIB HCC in the primary cohort who received long-term HPC (HPC time $>12$ minutes) had significantly better recurrence-free survival and overall survival than patients who received short-term HPC (HPC time $\leq 12$ minutes). (A) $P=0.007$. (B) $P=0.043$. 
Table 1: Univariate and multivariate analyses of recurrence-free survival (RFS) and overall survival (OS) of stage IIIB HCC patients in the primary cohort $(n=129)$

\begin{tabular}{|c|c|c|c|c|c|c|}
\hline \multirow{2}{*}{ Characteristics } & \multicolumn{3}{|c|}{ RFS } & \multicolumn{3}{|c|}{ OS } \\
\hline & $P$ value & HR & $95 \% \mathrm{CI}$ & $P$ value & HR & $95 \% \mathrm{CI}$ \\
\hline \multicolumn{7}{|l|}{ Univariate analysis } \\
\hline Sex: female vs. male & 0.205 & 1.254 & $0.883-1.781$ & 0.751 & 0.946 & $0.672-1.332$ \\
\hline Age: $>50$ vs. $\leq 50$ years & 0.331 & 0.721 & $0.491-1.054$ & 0.426 & 0.854 & $0.579-1.259$ \\
\hline HBsAg: positive vs. negative & 0.756 & 1.056 & $0.592-1.886$ & 0.811 & 1.076 & $0.589-1.964$ \\
\hline AFP: $>20$ vs. $\leq 20 \mathrm{ng} / \mathrm{ml}$ & 0.846 & 1.060 & $0.590-1.905$ & 0.372 & 1.268 & $0.753-2.134$ \\
\hline Liver cirrhosis: no vs. yes & 0.097 & 0.515 & $0.235-1.128$ & 0.180 & 0.606 & $0.292-1.261$ \\
\hline Tumour capsule: no vs. yes & 0.025 & 1.888 & $1.084-3.289$ & 0.500 & 1.096 & $0.840-1.431$ \\
\hline Tumour size: $>5$ vs. $\leq 5 \mathrm{~cm}$ & 0.029 & 1.467 & $1.264-3.429$ & 0.676 & 1.112 & $0.676-1.828$ \\
\hline Tumour number: single vs. multiple & 0.422 & 0.751 & $0.374-1.509$ & 0.260 & 1.339 & $0.805-2.228$ \\
\hline E-S Grading: I + II vs. III + IV & 0.145 & 1.433 & $0.883-2.326$ & 0.052 & 1.496 & $0.997-2.245$ \\
\hline HPC time: $>12$ vs. $\leq 12 \mathrm{~min}$ & 0.007 & 0.428 & $0.230-0.797$ & 0.043 & 0.609 & $0.376-0.985$ \\
\hline Surgical procedures: I vs. II§ & 0.357 & 0.607 & $0.210-1.755$ & 0.181 & 0.529 & $0.209-1.343$ \\
\hline Operation time: $\leq 165$ vs. $>165 \mathrm{~min}$ & 0.856 & 1.037 & $0.703-1.529$ & 0.634 & 0.908 & $0.611-1.350$ \\
\hline Intraoperative blood loss: $\leq 1.1$ vs. $>1.1 \mathrm{~L}$ & 0.853 & 1.046 & $0.650-1.683$ & 0.957 & 1.013 & $0.622-1.651$ \\
\hline Intraoperative blood transfusion: no vs. yes & 0.136 & 1.328 & $0.914-1.928$ & 0.412 & 1.175 & $0.799-1.726$ \\
\hline \multicolumn{7}{|l|}{ Multivariate analysis } \\
\hline Tumour capsule: no vs. yes & 0.013 & 2.019 & $1.157-3.523$ & NA & & \\
\hline Tumour size: $>5$ vs. $\leq 5 \mathrm{~cm}$ & 0.065 & 1.679 & $0.971-2.899$ & $\mathrm{NA}$ & & \\
\hline HPC time: $>12$ vs. $\leq 12 \mathrm{~min}$ & 0.005 & 0.410 & $0.220-0.765$ & $\mathrm{NA}$ & & \\
\hline
\end{tabular}

Abbreviations: $H C C$ hepatocellular carcinoma, $H R$ hazard ratio, $C I$ confidence interval, $H B s A g$ hepatitis B surface antigen, $A F P \alpha$-fetoprotein, $H P C$ hepatic pedicle clamping, $N A$ not analysed.

$\S$ Surgical procedures grade I: resection less than four liver segments. Surgical procedures grade II: resection of four or more liver segments.

\section{DISCUSSION}

The best treatment for advanced HCC including stage IIIB remains controversial and can lead to highrisk intrahepatic recurrence and low survival rate [16]. Furthermore, portal vein tumour thrombus probably occurs in $10-40 \%$ of patients at the time of diagnosis and in $44 \%$ of patients with $\mathrm{HCC}$ at the time of death [17]. The median survival of untreated HCC patients with $\mathrm{Vp} 4 / 3$ was reported to be 2.7 months [16]. Another largescale investigation of our institute revealed that the median survival of HCC patients with macroscopic portal vein tumour thrombus was 13.5 months [18]. In 2010, the Japan Society of Hepatology proposed that resection and transcatheter arterial chemoembolization (TACE) can be performed when there is portal invasion in HCC patients [19]. During hepatectomy, HPC was often applied to control blood loss to ensure a clear surgical field and to reduce the occurrence of postoperative liver failure [13]. In our study, there was no significant difference in surgical procedure, operation time, intraoperative blood loss, and blood transfusion between the LTHPC group and STHPC group. Thus, it cannot be argued that the possible negative effect was compensated by a decrease in operation time, intraoperative blood loss, and blood transfusion when HPC was used. It is common knowledge that HPC has long been used during elective hepatic resectional surgery to reduce operative blood loss and, in turn, decrease postoperative complications in hepatic tumour recurrence $[20,21]$. In the present study using a primary cohort and a validation cohort, we concluded for the first time that proper HPC application would improve both RFS and OS of stage IIIB HCC patients. The cut-off time of HPC in the present study was 12 minutes. However, we observed no significant impact of HPC on prognosis of all subjects, consistent with the previous report.

Yamanaka et al. reported that during hepatectomy, especially in patients with portal invasion, tumour cells would dislodge into the portal vein, and thus into the remnant liver, which would directly cause recurrence [22]. Similar results were also reported in pancreatic cancer, [23] lung cancer, [24] and colorectal cancer, [25] indicating that compression of tumours during operation would increase circulating tumor cell (CTC) numbers. It 
is predictable that $\mathrm{CTC}$ dissemination requires patency blood flow, which is blocked when HPC is adopted during hepatectomy. This may explain why HPC functioned as a protective factor in stage IIIB HCC patients.

However, I/R injury caused by HPC might have a negative impact on the prognosis. Several animal studies did demonstrate that $\mathrm{I} / \mathrm{R}$ injury caused by HPC would promote tumour growth and metastasis in both HCC and colorectal liver metastasis models [9, 26]. Results of clinical studies, however, were more complicated. Evidence presented by Yang et al. showed that selective main portal vein occlusion could minimise the risk

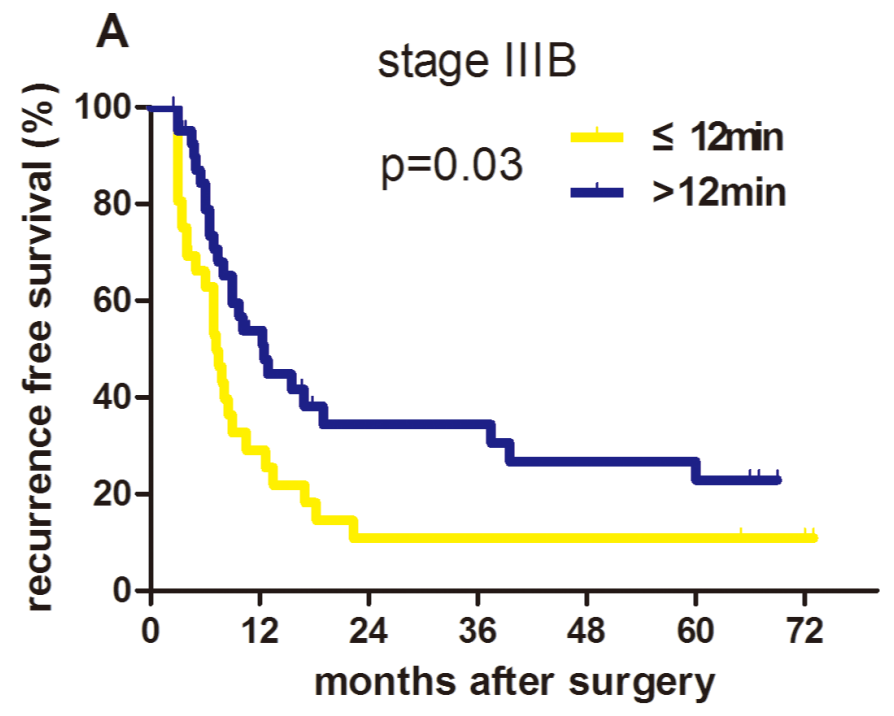

No. of patients at risk

HPC time (min)

$\begin{array}{lllllll}\leq 12 & 37 & 9 & 4 & 4 & 4 & 2 \\ >12 & 43 & 19 & 10 & 10 & 8 & 4\end{array}$

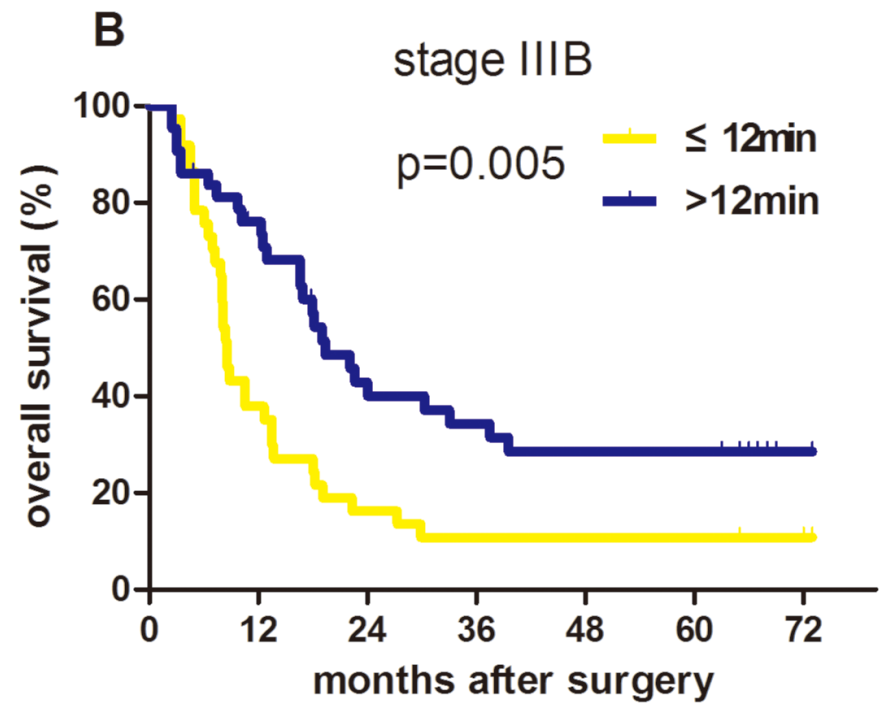

No. of patients at risk

HPC time (min)

$\begin{array}{lllccccc}\leq 12 & 37 & 16 & 7 & 5 & 5 & 5 & 3 \\ >12 & 43 & 30 & 16 & 13 & 11 & 11 & 5\end{array}$

Figure 2: Proper HPC application benefited the prognosis of stage IIIB HCC patients in the validation cohort. Patients with stage IIIB HCC in the validation cohort who received long-term HPC (HPC time $>12 \mathrm{~min}$ ) had significantly better recurrence-free survival and overall survival than patients who received short-time HPC (HPC time $\leq 12$ minutes). (A) $P=0.030$. (B) $P=0.005$. 
Table 2: Univariate and multivariate analyses of recurrence-free survival (RFS) and overall survival (OS) of stage IIIB HCC patients in the validation cohort $(n=80)$

\begin{tabular}{|c|c|c|c|c|c|c|}
\hline \multirow{2}{*}{ Characteristics } & \multicolumn{3}{|c|}{ RFS } & \multicolumn{3}{|c|}{ OS } \\
\hline & $P$ value & HR & $95 \% \mathrm{CI}$ & $P$ value & HR & $95 \% \mathrm{CI}$ \\
\hline \multicolumn{7}{|l|}{ Univariate analysis } \\
\hline Sex: female vs. male & 0.521 & 1.466 & $0.455-4.723$ & 0.875 & 1.098 & $0.343-3.509$ \\
\hline Age: $>50$ vs. $\leq 50$ years & 0.207 & 1.425 & $0.822-2.47$ & 0.180 & 1.433 & $0.847-2.425$ \\
\hline HBsAg: positive vs. negative & 0.976 & 1.010 & $0.52-1.964$ & 0.184 & 1.591 & $0.802-3.157$ \\
\hline AFP: $>20$ vs. $\leq 20 \mathrm{ng} / \mathrm{ml}$ & 0.209 & 1.455 & $0.81-2.615$ & 0.012 & 2.122 & $1.178-3.824$ \\
\hline Liver cirrhosis: no vs. yes & 0.284 & 0.604 & $0.24-1.519$ & 0.298 & 0.615 & $0.246-1.538$ \\
\hline Tumour capsule: no vs. yes & 0.605 & 1.200 & $0.602-2.392$ & 0.867 & 1.057 & $0.55-2.034$ \\
\hline Tumour size: $>5$ vs. $\leq 5 \mathrm{~cm}$ & 0.764 & 1.101 & $0.586-2.069$ & 0.032 & 2.06 & $1.064-3.987$ \\
\hline Tumour number: single vs. multiple & 0.851 & 1.08 & $0.486-2.397$ & 0.811 & 1.102 & $0.498-2.435$ \\
\hline E-S Grading: I + II vs. III + IV & 0.582 & 0.858 & $0.498-1.479$ & 0.816 & 1.062 & $0.639-1.765$ \\
\hline HPC time: $>12$ vs. $\leq 12 \mathrm{~min}$ & 0.030 & 0.551 & $0.322-0.945$ & 0.005 & 0.473 & $0.283-0.793$ \\
\hline Surgical procedures: I vs. II§ & 0.235 & 0.723 & $0.423-1.235$ & 0.847 & 1.057 & $0.602-1.856$ \\
\hline Operation time: $\leq 165$ vs. $>165 \mathrm{~min}$ & 0.912 & 1.028 & $0.634-1.666$ & 0.947 & 0.983 & $0.592-1.633$ \\
\hline Intraoperative blood loss: $\leq 1.1$ vs. $>1.1 \mathrm{~L}$ & 0.590 & 0.831 & $0.423-1.631$ & 0.422 & 1.382 & $0.627-3.045$ \\
\hline Intraoperative blood transfusion: no vs. yes & 0.689 & 0.898 & $0.531-1.520$ & 0.748 & 1.096 & $0.625-1.923$ \\
\hline \multicolumn{7}{|l|}{ Multivariate analysis } \\
\hline AFP: $>20$ vs. $\leq 20 \mathrm{ng} / \mathrm{ml}$ & NA & & & 0.157 & 1.584 & $0.838-2.994$ \\
\hline Tumour size: $>5$ vs. $\leq 5 \mathrm{~cm}$ & NA & & & 0.179 & 1.616 & $0.802-3.257$ \\
\hline HPC time: $>12$ vs. $\leq 12 \mathrm{~min}$ & NA & & & 0.019 & 0.535 & $0.317-0.904$ \\
\hline
\end{tabular}

Abbreviations: $H C C$ hepatocellular carcinoma, $H R$ hazard ratio, $C I$ confidence interval, $H B s A g$ hepatitis B surface antigen, $A F P \alpha$-fetoprotein, $H P C$ hepatic pedicle clamping, $N A$ not analysed.

$\S$ Surgical procedures grade I: resection less than four liver segments. Surgical procedures grade II: resection of four or more liver segments.

of recurrence after curative resection of HCC [15]. Recently, a case-matched study involving colorectal liver metastasis patients figured that the 5-year recurrencefree rate of HPC patients was significantly higher than that of patients who did not receive HPC [27]. However, it was rather remarkable that another study conducted by Xia et al. described no significant results between HCC patients undergoing HPC or not during hepatectomy [28]. Other studies also observed no significant impact of HPC on the prognosis of patients with colorectal liver metastasis [29-33]. In the present study, no significant impact of HPC on the prognosis of all subjects was observed, consistent with previous reports by Xia et al. Interestingly, Makino et al. [28]. reported that selective portal vein occlusion, compared with total portal vein occlusion, during hepatic resection allowed better prognosis for HCC patients [14]. In this study, better prognosis was only shown in the selective portal vein occlusion (SPVO) group, in which blood flow was blocked in the tumour-bearing side with minimal $\mathrm{I} / \mathrm{R}$ injury of the remnant liver. Moreover, the SPVO group also had reduced impact of tumour cell dissemination. Because there was no consideration of CTC dissemination, this might explain the high rate of metastasis and recurrence in HCC animal models caused by HPC-induced I/R injury. Only when CTC dissemination and I/R injury were taken into consideration could we exactly evaluate the role of HPC in HCC prognosis. Another study reported by Tanaka et al. might provide great evidence for the interaction of CTC dissemination and I/R injury. They showed that HPC during hepatectomy significantly reduced postoperative metastasis to extrahepatic sites, whereas no significance was observed for intrahepatic recurrence [34].

This study has several inherent limitation that should be addressed. It's a retrospective study to discuss the effects of HPC on patients' outcomes after hepatic resection. The findings may limited by selection bias and confounding factors, which could be minimize a prospective randomized controlled study. It may lead to selection bias because of the use of HPC mainly depending on the individual surgeons' preference during operation. The two cohorts including primary cohort and validation cohort may reduce the selection bias and the confounding factors. 
To our knowledge, we were the first to conclude that although HPC had no impact on the prognosis of all stages of I-IIIA HCC patients, consistent with the previous results reported by Xia et al., [28] proper application of HPC was a protective factor for stage IIIB HCC patients who were suffering from macroscopic vascular invasion. Future studies should be conducted to determine if HPC during hepatectomy will reduce tumour cell dissemination, to clarify the interaction between tumour cell dissemination and $\mathrm{I} / \mathrm{R}$ injury, and to confirm whether selective main portal vein occlusion [15] or portal vein clamping [14] would be more positive for the prognosis of stage IIIB HCC patients. In the present study, we found that proper HPC did improve the postoperative prognosis of stage IIIB HCC patients. We proposed that if the parenchymal liver resection time was preoperatively evaluated over 12 minutes, then proper HPC should be performed during hepatectomy for improvement in prognosis.

\section{MATERIALS AND METHODS}

\section{Patients}

Tumour stages were determined by the 2010 American Joint Committee on Cancer (AJCC) 7th TNM staging system [35]. From January 2003 to December
2006, 2527 consecutive patients underwent liver resection by the same medical center. The status of disease and resectability were assessed by contrast-enhanced magnetic imaging, ultrasonography, and computed tomography. 1401 HCC patients with stage I/II/IIIA/IIIB HCC undergoing tumour resection in Zhongshan Hospital were retrospectively included in the primary cohort of the present study; 80 stage IIIB HCC patients underwent curative resection from January 2007 to December 2007 were included in the validation cohort (Figure 3). This study was approved by the Research Ethics Committee of Zhongshan Hospital, and informed consent was obtained from all subjects.

The inclusion criteria were as follows: (1) HCC diagnosed pathologically after resection and (2) patients underwent curative resection for $\mathrm{HCC}$, defined as complete macroscopic removal of the tumour and cancerous thrombus. The exclusion criteria were as follows: (1) patients with distant metastasis or local organ invasion; (2) patients with other origins of cancer previously; (3) patients who died within 30 days after surgery; (4) total HPC time exceeded 40 minutes or HPC was used more than twice during the operation; (5) patients received other treatment prior to liver resection; and (6) patients were lost at the beginning of the follow-up.

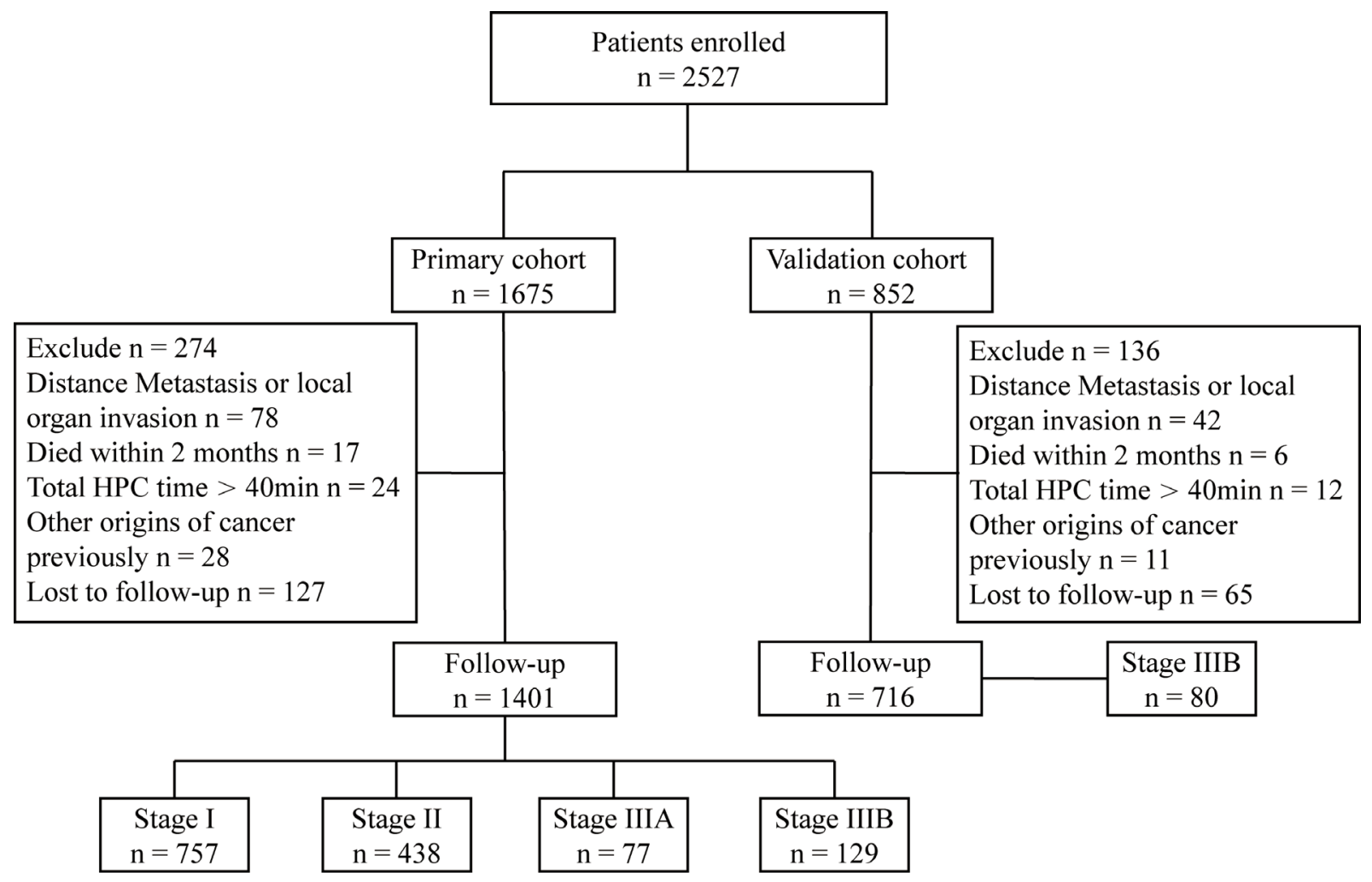

Figure 3: Study design. From January 2003 to December 2006, 1401 patients undergoing tumour resection in Zhongshan Hospital were retrospectively included in the primary cohort, which consisted of 757 stage I, 438 stage II, 77 stage IIIA, and 129 stage IIIB HCC patients. The primary cohort was used to determine the baseline HPC time and investigate the impact of HPC time on prognosis. Another 80 stage IIIB HCC patients also in Zhongshan Hospital from January 2007 to December 2007 were included in the validation cohort. This cohort was used to verify the improvement of the prognosis with proper HPC in stage IIIB HCC patients. 
All patients were monitored by serum alphafetoprotein (AFP), abdomen ultrasonography, and chest $\mathrm{X}$-ray every 1 to 6 months and magnetic resonance imaging (MRI) every 6 to 12 months according to the postoperative time. For patients with test results suggestive of recurrence, computed tomography and/or magnetic resonance imaging were used to verify whether recurrence had occurred. The diagnosis of recurrence was based on the combination of imaging and clinical evaluations.

Patients lost to follow-up were censored at their last encounter. Mortality was defined as death within the first 30 postoperative days, and these patients were excluded as described in the exclusion criteria.

\section{Clinicopathological factors}

Clinicopathological factors in this study were selected for their potential relation to the prognosis on the basis of the previous studies [36, 37], including age (50 years or younger or older than 50 years), gender (male or female), HBsAg status (positive or negative), liver cirrhosis (yes or no), serum AFP concentration $(\leq 20$ or $>20 \mathrm{ng} / \mathrm{mL})$, tumour size $(\leq 5$ or $>5 \mathrm{~cm})$, number of tumour nodules (solitary or multiple), tumour capsule (positive or negative), and differentiation of tumour cells (Edmondson's classification I/II or III/IV) [38]. The characteristics regarding how surgical outcomes may affect the prognosis were also selected based on the previous study, including surgical procedure ( $I, \leq 3$ segments; II, > 3 segments), and based on Couinaud's nomenclature [39], operation time, intraoperative blood loss, and intraoperative blood transfusion (yes or no). For the laboratory parameters, the cutoff values were the upper limit of the normal values in our hospital.

\section{Treatment}

Liver resection included nonanatomical resection, subsegmentectomy, segmentectomy, hemihepatectomy, and trisecmentectomy. A major hepatectomy is defined as resection of four or more Couinaud's segments. The extent of hepatectomy was evaluated according to the extent of disease progression, liver function, and general status of patients. HPC (i.e., Pringle manoeuver), if used, was achieved by tightening 4-mm tape around the portal triad. Intermittent HPC consisted of cycles of 15-20 min clamping followed by 5 minutes unclamping. The use of HPC was based on the individual preference of surgeons during operation. The time of HPC was recorded by the nurse.

\section{Statistics}

The RFS time was defined as the interval between the operation and the date of diagnosis of the first recurrence. The OS time was defined as the interval between the operation and death. The optimal cut-off time of HPC was assessed by the receiver-operator characteristic (ROC) curve and estimated the area under the curve (using the calculator for ROC curves available from Johns Hopkins University, Baltimore, MD, USA [www.jrocfit.org, accessed 30 April 2008]). Each point on the ROC curve represents a sensitivity/specificity pair corresponding to a particular decision threshold. A best test shows the ROC curve that passes through the upper left-hand corner (100\% sensitivity, $100 \%$ specificity)[40-42]. Therefore the larger area under the ROC curve means the higher overall accuracy of the test. The values yielding maximum sums from the ROC curves got the best sensitivity and specificity. Associations between HPC time and patient characteristics were analyzed using the $\chi^{2}$ test (or Fisher's exact test) for two categorical variables. Survival curves were calculated by the KaplanMeier method and compared by log-rank test. Multivariate analysis was performed using Cox proportional hazards model. Stratified log-rank tests were used to test the effect of HPC whilst controlling for the effect of another variable. Variables with $P<0.05$ on univariate analysis were further evaluated by multivariate analysis.

\section{ACKNOWLEDGMENTS}

We thank Dr. Chaoqun Wang and Haoting Sun of Department of General Surgery, Huashan Hospital, Fudan University for helping to analyze the data. This work was supported in part by grants from the State Key Basic Research Program of China (2013CB910500), the National Key Project for Infectious Disease of China (2012ZX10002012-003), and the National Natural Science Foundation of China (81071993, 81372654).

\section{CONFLICTS OF INTEREST}

The authors declare no conflicts of interest.

\section{REFERENCES}

1. Torre LA, Bray F, Siegel RL, Ferlay J, Lortet-Tieulent J, Jemal A. Global cancer statistics, 2012. CA Cancer J Clin. 2015; 65:87-108.

2. Mittal S, El-Serag HB. Epidemiology of hepatocellular carcinoma: consider the population. Journal of clinical gastroenterology. 2013; 47:S2-6.

3. European Association For The Study Of The Liver; European Organisation For Research And Treatment Of Cancer. EASL-EORTC clinical practice guidelines: management of hepatocellular carcinoma. European journal of cancer. 2012; 48:599-641.

4. Lencioni R. Loco-regional treatment of hepatocellular carcinoma. Hepatology. 2010; 52:762-773.

5. Tabrizian P, Schwartz ME. Surgical management of hepatocellular carcinoma. The Mount Sinai journal of medicine. 2012; 79:223-231. 
6. Kishi Y, Hasegawa K, Sugawara Y, Kokudo N. Hepatocellular carcinoma: current management and future development-improved outcomes with surgical resection. International journal of hepatology. 2011; 2011:728103.

7. Bruix J, Gores GJ, Mazzaferro V. Hepatocellular carcinoma: clinical frontiers and perspectives. Gut. 2014; 63:844-855.

8. Yin J, Li N, Han Y, Xue J, Deng Y, Shi J, Guo W, Zhang H, Wang H, Cheng S, Cao G. Effect of antiviral treatment with nucleotide/nucleoside analogs on postoperative prognosis of hepatitis B virus-related hepatocellular carcinoma: a two-stage longitudinal clinical study. Journal of clinical oncology. 2013; 31:3647-3655.

9. Man K, Ng KT, Lo CM, Ho JW, Sun BS, Sun CK, Lee TK, Poon RT, Fan ST. Ischemia-reperfusion of small liver remnant promotes liver tumor growth and metastasesactivation of cell invasion and migration pathways. Liver transplantation. 2007; 13:1669-1677.

10. Doi K, Horiuchi T, Uchinami M, Tabo T, Kimura N, Yokomachi J, Yoshida M, Tanaka K. Neutrophil elastase inhibitor reduces hepatic metastases induced by ischaemiareperfusion in rats. The European journal of surgery. 2002; 168:507-510.

11. van der Bilt JD, Soeters ME, Duyverman AM, Nijkamp MW, Witteveen PO, van Diest PJ, Kranenburg O, Borel Rinkes IH. Perinecrotic hypoxia contributes to ischemia/reperfusion-accelerated outgrowth of colorectal micrometastases. The American journal of pathology. 2007; 170:1379-1388.

12. Lim C, Broqueres-You D, Brouland JP, Merkulova-Rainon T, Faussat AM, Hilal R, Rouquie D, Eveno C, Audollent R, Levy BI, Pocard M. Hepatic ischemia-reperfusion increases circulating bone marrow-derived progenitor cells and tumor growth in a mouse model of colorectal liver metastases. The Journal of surgical research. 2013; 184:888-897.

13. Dixon E, Vollmer CM, Jr., Bathe OF, Sutherland F. Vascular occlusion to decrease blood loss during hepatic resection. American journal of surgery. 2005; 190:75-86.

14. Makino I, Chijiiwa K, Kondo K, Ohuchida J, Kai M. Prognostic benefit of selective portal vein occlusion during hepatic resection for hepatocellular carcinoma. Surgery. 2005; 137:626-631.

15. Yang Y, Fu SY, Lau WY, Lai E, Li AJ, Pan ZY, Zhou WP, Shen F, Wu MC. Selective main portal vein clamping to minimize the risk of recurrence after curative liver resection for hepatocellular carcinoma. Hepato-gastroenterology. 2012; 59:1560-1565.

16. Yoshidome H, Takeuchi D, Kimura F, Shimizu H, Ohtsuka M, Kato A, Furukawa K, Yoshitomi H, Miyazaki M. Treatment strategy for hepatocellular carcinoma with major portal vein or inferior vena cava invasion: a single institution experience. Journal of the American College of Surgeons. 2011; 212:796-803.

17. Zhu K, Chen J, Lai L, Meng X, Zhou B, Huang W, Cai M, Shan H. Hepatocellular carcinoma with portal vein tumor thrombus: treatment with transarterial chemoembolization combined with sorafenib - a retrospective controlled study. Radiology. 2014; 272:284-293.

18. Fan J, Tang ZY, Wu ZQ, Zhou J, Zhou XD, Ma ZC, Qin LX, Qiu SJ, Yu Y, Huang C. The effects of portal vein microscopic and macroscopic tumor thrombi on postoperation patients with hepatocellular carcinoma [Article in Chinese]. Zhonghua wai ke za zhi. 2005; 43:433-435.

19. Kudo M, Ueshima K. Positioning of a moleculartargeted agent, sorafenib, in the treatment algorithm for hepatocellular carcinoma and implication of many complete remission cases in Japan. Oncology. 2010; 78:154-166.

20. Benoist S, Salabert AS, Penna C, Karoui M, Julie C, Rougier P, Nordlinger B. Portal triad clamping (TC) or hepatic vascular exclusion (VE) for major liver resection after prolonged neoadjuvant chemotherapy? A casematched study in 60 patients. Surgery. 2006; 140:396-403.

21. Ercolani G, Ravaioli M, Grazi GL, Cescon M, Del Gaudio M, Vetrone G, Zanello M, Pinna AD. Use of vascular clamping in hepatic surgery: lessons learned from 1260 liver resections. Archives of surgery. 2008; 143:380-387; discussion 388.

22. Yamanaka N, Okamoto E, Fujihara S, Kato T, Fujimoto J, Oriyama T, Mitsunobu M, Toyosaka A, Uematsu K, Yamamoto K. Do the tumor cells of hepatocellular carcinomas dislodge into the portal venous stream during hepatic resection? Cancer. 1992; 70:2263-2267.

23. Gall TM, Jacob J, Frampton AE, Krell J, Kyriakides C, Castellano L, Stebbing J, Jiao LR. Reduced dissemination of circulating tumor cells with no-touch isolation surgical technique in patients with pancreatic cancer. JAMA surgery. 2014; 149:482-485.

24. Hashimoto M, Tanaka F, Yoneda K, Takuwa T, Matsumoto S, Okumura Y, Kondo N, Tsubota N, Tsujimura T, Tabata C, Nakano T, Hasegawa S. Significant increase in circulating tumour cells in pulmonary venous blood during surgical manipulation in patients with primary lung cancer. Interactive cardiovascular and thoracic surgery. 2014; 18:775-783.

25. Takii Y, Shimada Y, Moriya Y, Nakamura K, Katayama H, Kimura A, Shibata T, Fukuda H, Colorectal Cancer Study Group of Japan Clinical Oncology G. A randomized controlled trial of the conventional technique versus the no-touch isolation technique for primary tumor resection in patients with colorectal cancer: Japan Clinical Oncology Group Study JCOG1006. Japanese journal of clinical oncology. 2014; 44:97-100.

26. Tamagawa K, Horiuchi T, Uchinami M, Doi K, Yoshida M, Nakamura T, Sasaki H, Taniguchi M, Tanaka K. Hepatic ischemia-reperfusion increases vascular endothelial growth factor and cancer growth in rats. The Journal of surgical research. 2008; 148:158-163.

27. De Carlis L, Di Sandro S, Giacomoni A, Mihaylov P, Lauterio A, Mangoni I, Cusumano C, Poli C, Tripepi M, Bencardino K. Colorectal liver metastases: hepatic pedicle 
clamping during hepatectomy reduces the incidence of tumor recurrence in selected patients. Case-matched analysis. European journal of surgical oncology. 2013; 39:726-733.

28. Xia F, Lau WY, Xu Y, Wu L, Qian C, Bie P. Does hepatic ischemia-reperfusion injury induced by hepatic pedicle clamping affect survival after partial hepatectomy for hepatocellular carcinoma? World journal of surgery. 2013; 37:192-201.

29. Wong KH, Hamady ZZ, Malik HZ, Prasad R, Lodge JP, Toogood GJ. Intermittent Pringle manoeuvre is not associated with adverse long-term prognosis after resection for colorectal liver metastases. The British journal of surgery. 2008; 95:985-989.

30. Ferrero A, Russolillo N, Vigano L, Lo Tesoriere R, Muratore A, Capussotti L. Does Pringle maneuver affect survival in patients with colorectal liver metastases? World journal of surgery. 2010; 34:2418-2425.

31. Giuliante F, Ardito F, Pulitano C, Vellone M, Giovannini I, Aldrighetti L, Ferla G, Nuzzo G. Does hepatic pedicle clamping affect disease-free survival following liver resection for colorectal metastases? Annals of surgery. 2010; 252:1020-1026.

32. Matsuda A, Miyashita M, Matsumoto S, Matsutani T, Sakurazawa N, Akagi I, Kishi T, Yokoi K, Uchida E. Hepatic pedicle clamping does not worsen survival after hepatic resection for colorectal liver metastasis: results from a systematic review and meta-analysis. Annals of surgical oncology. 2013; 20:3771-3778.

33. Weiss $\mathrm{MJ}$, Ito $\mathrm{H}$, Araujo $\mathrm{RL}$, Zabor $\mathrm{EC}$, Gonen $\mathrm{M}$, D'Angelica MI, Allen PJ, DeMatteo RP, Fong Y, Blumgart LH, Jarnagin WR. Hepatic pedicle clamping during hepatic resection for colorectal liver metastases: no impact on survival or hepatic recurrence. Annals of surgical oncology. 2013; 20:285-294.
34. Tanaka K, Shimada H, Matsuo K, Takeda K, Nagano Y, Togo S. Clinical features of hepatocellular carcinoma developing extrahepatic recurrences after curative resection. World journal of surgery. 2008; 32:1738-1747.

35. Edge S, Byrd DR, Compton, C.C., Fritz, A.G., Greene, F.L., Trotti A and (Eds.). AJCC Cancer Staging Manual (ed 7th). New York, NY, Springer. 2010; 191-195.

36. Gao Q, Qiu SJ, Fan J, Zhou J, Wang XY, Xiao YS, Xu Y, Li YW, Tang ZY. Intratumoral balance of regulatory and cytotoxic $\mathrm{T}$ cells is associated with prognosis of hepatocellular carcinoma after resection. Journal of clinical oncology. 2007; 25:2586-2593.

37. Zhu XD, Zhang JB, Zhuang PY, Zhu HG, Zhang W, Xiong YQ, Wu WZ, Wang L, Tang ZY, Sun HC. High expression of macrophage colony-stimulating factor in peritumoral liver tissue is associated with poor survival after curative resection of hepatocellular carcinoma. Journal of clinical oncology. 2008; 26:2707-2716.

38. Edmondson HA, Steiner PE. Primary carcinoma of the liver: a study of 100 cases among 48,900 necropsies. Cancer. 1954; 7:462-503.

39. Couinaud C. [The anatomy of the liver]. Annali italiani di chirurgia. 1992; 63:693-697.

40. Metz CE. Basic principles of ROC analysis. Seminars in nuclear medicine. 1978; 8:283-298.

41. Van der Schouw YT, Verbeek AL, Ruijs JH. ROC curves for the initial assessment of new diagnostic tests. Family practice. 1992; 9:506-511.

42. Zweig MH, Campbell G. Receiver-operating characteristic (ROC) plots: a fundamental evaluation tool in clinical medicine. Clinical chemistry. 1993; 39:561-577. 